\title{
Direct observation of negative thermal expansion in $\mathrm{SrCu}_{3} \mathrm{Fe}_{4} \mathrm{O}_{12}$
}

\author{
Ikuya YAMADA, ${ }^{*, * * \dagger}$ Kentaro SHIRO,${ }^{* * *}$ Kengo OKA, ${ }^{* * * *}$ Masaki AZUMA $^{* * * *}$ and Tetsuo IRIFUNE ${ }^{* * * * *}$ \\ *Nanoscience and Nanotechnology Research Center, Osaka Prefecture University, \\ 1-2 Gakuen-cho, Naka-ku, Sakai 599-8570, Japan \\ **PRESTO, Japan Science and Technology Agency (JST), 4-1-8 Honmachi, Kawaguchi, Saitama 332-0012, Japan \\ ${ }^{* * *}$ Department of Chemistry, Graduate School of Science and Engineering, Ehime University, \\ 2-5 Bunkyo-cho, Matsuyama 790-8577, Japan \\ ${ }^{* * * * *}$ Materials and Structures Laboratory, Tokyo Institute of Technology, Nagatsuta, Midori, Yokohama 226-8503, Japan \\ ***** Geodynamics Research Center (GRC), Ehime University, 2-5 Bunkyo-cho, Matsuyama 790-8577, Japan
}

We report a direct observation of the negative thermal expansion in an iron-based perovskite oxide $\mathrm{SrCu}_{3} \mathrm{Fe}_{4} \mathrm{O}_{12}$ by using a thermomechanical analyzer and strain gauge. Reversibility and small thermal hysteresis in the negative thermal expansion temperature range demonstrate that the negative thermal expansion of this compound is attributed to second-order phase transition.

(C2013 The Ceramic Society of Japan. All rights reserved.

Key-words : Negative thermal expansion, Perovskite, Unusual high valence ion, High-pressure synthesis, Dilatometric analysis

\section{Introduction}

Negative thermal expansion (NTE) materials, which show volume contraction upon warming (volume expansion upon cooling), have been extensively investigated because of their potential utility in precision mechanical engineering and optics. ${ }^{1), 2)}$ Combination of NTE materials with conventional materials having a positive thermal expansion may help suppress degradation resulting from volume expansion and contraction during thermal cycles and aid in precise control of the operating life. Recently, several novel NTE materials with large negative coefficients of linear thermal expansion (CTE, $\left.\alpha_{L}\right)$, on the order of $10^{-5} \mathrm{~K}^{-1}$, were reported. For instance, antiperovskitetype nitrides $\mathrm{Mn}_{3}(\mathrm{Cu}, \mathrm{Ge}) \mathrm{N}$ showed a large NTE $\left(\alpha_{L}=-1.6 \times\right.$ $10^{-5} \mathrm{~K}^{-1}$ ) near room temperature, which was achieved by relaxation of the magnetovolume effect of the parent compound $\mathrm{Mn}_{3} \mathrm{CuN}^{3)}$ A perovskite-type oxide $(\mathrm{Bi}, \mathrm{La}) \mathrm{NiO}_{3}$ exhibited a significantly high NTE $\left(\alpha_{L}=-8.2 \times 10^{-5} \mathrm{~K}^{-1}\right)$ above room temperature because of the first-order phase transition temperature, at which intersite charge transfer (simplified as $\mathrm{Bi}^{4+}+$ $\mathrm{Ni}^{2+} \rightarrow \mathrm{Bi}^{3+}+\mathrm{Ni}^{3+}$ on heating) is dominant. ${ }^{4}$

$\mathrm{SrCu}_{3} \mathrm{Fe}_{4} \mathrm{O}_{12}$ (SCFO) belongs to the class of cubic A-site ordered perovskites $\mathrm{ACu}_{3} \mathrm{Fe}_{4} \mathrm{O}_{12}(\mathrm{~A}=\mathrm{Ca}, \mathrm{Sr}, \mathrm{La} \text {, and } \mathrm{Y})^{5)-8)}$ with 1:3-type ordering of the A-site cations (see the crystal structure in Fig. 1). ${ }^{9)}$ Coexistence of the two transition metal ( $\mathrm{Cu}$ and $\mathrm{Fe}$ ) ions at different crystallographic sites in $\mathrm{ACu}_{3} \mathrm{Fe}_{4} \mathrm{O}_{12}$ induces intriguing electronic interactions between these ions, such as intersite charge transfer and magnetic superexchange interactions. Rietveld refinement of SCFO based on synchrotron X-ray powder diffraction (SXRD) revealed drastic changes in metal-oxygen bond lengths, that is, gradual elongation (shrinkage) of the $\mathrm{Cu}-\mathrm{O}$ (Fe-O) bond upon heating in the temperature range 170-270 K, leading to an NTE $\left(\alpha_{L}=-2.3 \times 10^{-5} \mathrm{~K}^{-1}\right){ }^{6}{ }^{6}$ Bond valence sum calculations suggested that the above-mentioned structural trans-

Corresponding author: I. Yamada; E-mail: i-yamada@21c. osakafu-u.ac.jp

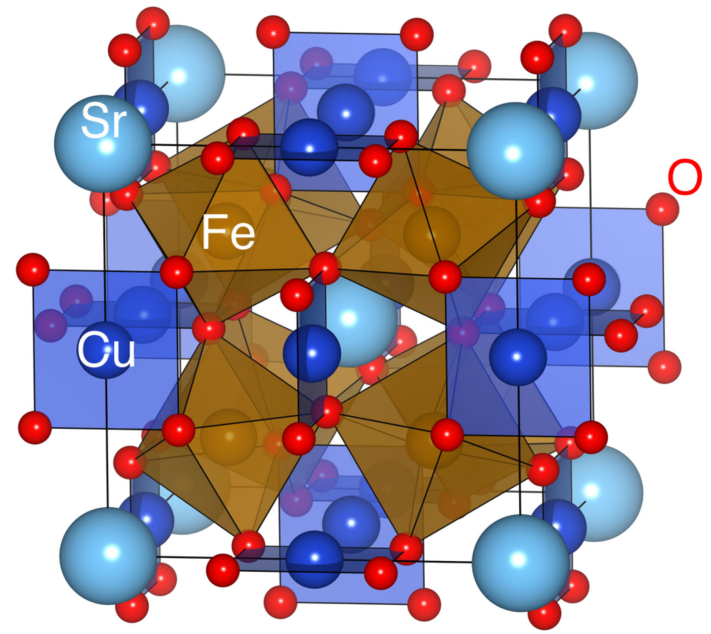

Fig. 1. Crystal structure of $\mathrm{SrCu}_{3} \mathrm{Fe}_{4} \mathrm{O}_{12}$. The light-blue, dark-blue, brown, and red spheres represent $\mathrm{Sr}, \mathrm{Cu}, \mathrm{Fe}$, and $\mathrm{O}$ atoms, respectively.

formations correspond to a crossover-like intersite charge transfer between the $\mathrm{Cu}$ and $\mathrm{Fe}$ ions $\left(\mathrm{Cu}^{3+}+\mathrm{Fe}^{3+} \rightarrow \mathrm{Cu}^{2+}+\mathrm{Fe}^{4+}\right.$ on heating), resulting in an NTE in this temperature range.

In this study, we show a direct observation of the NTE of SCFO by dilatometric analysis. The dilatometric data demonstrated a negative CTE of $\alpha_{L}=-7 \times 10^{-6} \mathrm{~K}^{-1}$. Reversibility and significantly small thermal hysteresis in the NTE temperature range were also confirmed by the dilatometric measurements.

\section{Experimental procedure}

The precursor of $\mathrm{SrCu}_{3} \mathrm{Fe}_{4} \mathrm{O}_{12}$ was prepared by the polymerized complex method in the same manner described in a previous report. ${ }^{6}$ The precursor powder with a nominal formula of $\mathrm{SrCu}_{3}$ $\mathrm{Fe}_{4} \mathrm{O}_{10}$ was mixed with $\mathrm{KClO}_{4}(99.9 \%)$ in a molar ratio of $2: 1$ in order to compensate for the oxygen stoichiometry. The mixture was compressed to $15 \mathrm{GPa}$ using a Kawai-type high-pressure 
apparatus, heated to $1273 \mathrm{~K}$ for $30 \mathrm{~min}$, maintained at this temperature for $30 \mathrm{~min}$, and quenched to ambient temperature. The pressure was slowly released after the heat treatment. The obtained powder was washed with water to remove the residual $\mathrm{KCl}$. Pelletized samples for dilatometric measurements were prepared by sintering the above-mentioned powder at $15 \mathrm{GPa}$ and $1273 \mathrm{~K}$ for $30 \mathrm{~min}$. The obtained pellets were $\sim 2 \mathrm{~mm}$ in diameter and $1-2 \mathrm{~mm}$ in height.

$\mathrm{X}$-ray powder diffraction (XRD) patterns were collected over the temperature range $100-450 \mathrm{~K}$ with a $10 \mathrm{~K}$ step, in a RIGAKU Ultima IV X-ray diffractometer using $\mathrm{Cu} \mathrm{K} \alpha$ radiation. Synchrotron X-ray diffraction (SXRD) pattern at room temperature was collected using a Debye-Scherrer camera installed at the BL02B2 beamline of SPring-8. Lattice constants and phase fraction were refined by Rietveld refinement using the program RIETAN-FP. ${ }^{10)}$ Linear thermal expansion of the sintered pellets was measured by means of two kinds of dilatometric methods. Thermomechanical analysis (TMA) measurements were conducted over the temperature range $125-400 \mathrm{~K}$ using a thermomechanical analizer RIGAKU TMA8310 system. Strain gauge (SG) measurements were performed in the temperature range $4-400 \mathrm{~K}$ using a KYOWA-type KFL strain gauge on a He refrigerator PASCAL 101D HE. The cooling/heating rate in the TMA and SG measurements were $\sim 3.1$ and $\sim 1.6 \mathrm{~K} / \mathrm{s}$, respectively.

\section{Results and discussion}

Figure 2 shows the room-temperature SXRD pattern and Rietveld refinement result of the sample prepared by grinding the sintered pellet after the TMA and SG measurements. The reliability factors $\left(R_{\mathrm{wp}}=3.692 \%, R_{\mathrm{B}}=1.699 \%\right)$ and goodnessof-fit $(S=1.0025)$ indicate that the refinement result is appropriate. The primary phase could be indexed in the cubic A-site ordered perovskite structure with the space group $\operatorname{Im} \overline{3}$. The refined value of the lattice constant $a$ was 7.36053(19) $\AA$, which roughly agreed with that mentioned in a previous report (7.34921 $\mathrm{A}) .{ }^{6)}$ Several impurities such as $\mathrm{CuO}, \alpha-\mathrm{Fe}_{2} \mathrm{O}_{3}$, and unknown phase(s) were observed (see the inset of Fig. 2). The volume fractions of $\mathrm{SCFO}, \mathrm{CuO}$, and $\alpha-\mathrm{Fe}_{2} \mathrm{O}_{3}$ estimated from the Rietveld refinement were $71.14,15.45$, and $13.42 \%$, respectively.

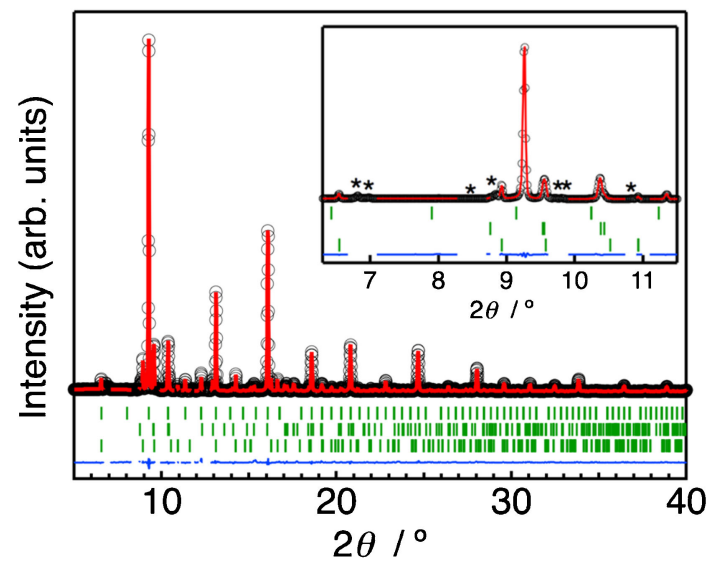

Fig. 2. XRD pattern of $\mathrm{SrCu}_{3} \mathrm{Fe}_{4} \mathrm{O}_{12}$ measured at room temperature. The black circles and red line represent the observed and calculated profiles, respectively., The blue line in the bottom shows the difference between the observation and calculation. The vertical bars display the Bragg reflection positions for $\mathrm{SrCu}_{3} \mathrm{Fe}_{4} \mathrm{O}_{12}, \mathrm{CuO}$, and $\alpha-\mathrm{Fe}_{2} \mathrm{O}_{3}$ from top to bottom, respectively. The inset shows the enlarged pattern in the selected $2 \theta$ range $\left(6.3-11.5^{\circ}\right)$. The asterisks represent the Bragg reflection positions for unknown phases.
Figure 3(a) shows the temperature dependence of the linear thermal expansion $\mathrm{d} L / L_{400 \mathrm{~K}}$ of SCFO, as determined by SXRD (data from Ref. 6), XRD, TMA, and SG measurements. The XRD data in this study show an NTE at about the same temperature range as the SXRD data. The CTE, obtained from a linear fitting of the XRD data between 230 and $270 \mathrm{~K}$, was $-2.3 \times$ $10^{-5} \mathrm{~K}^{-1}$ [see Fig. 3(b)], which is consistent with a previous report. $\left.{ }^{6}\right)$ The continuous change in the linear thermal expansion suggested the second-order-type phase transformation. The TMA and SG data also demonstrated an NTE over a similar temperature range as the SXRD and XRD, which are direct observations of the NTE for SCFO. The magnitude of the NTE was smaller than that obtained from the SXRD and XRD data; the $\alpha_{L}$ values were calculated as $-7.4 \times 10^{-6}$ and $-6.5 \times 10^{-6} \mathrm{~K}^{-1}$ from the TMA and SG data, respectively. This is mainly because the TMA

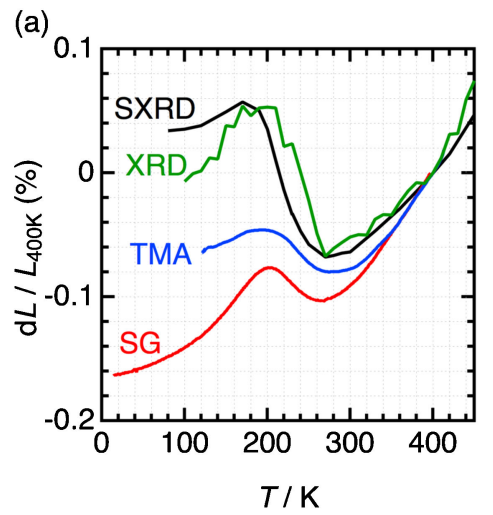

(b)

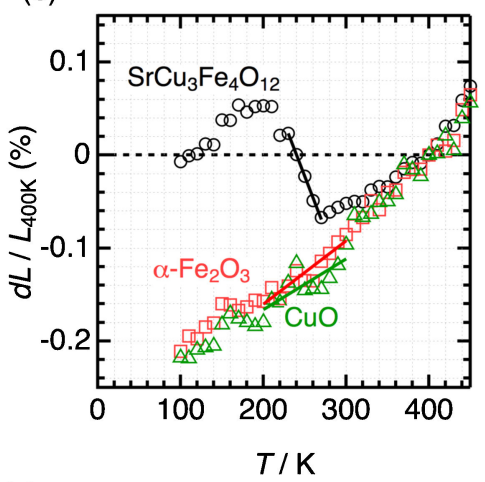

(c)

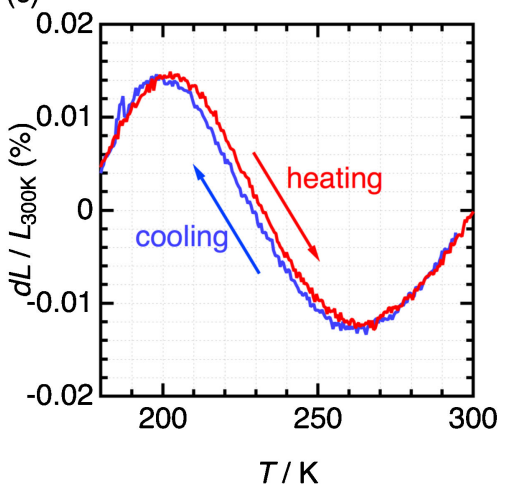

Fig. 3. (a) Linear thermal expansion $\mathrm{d} L / L_{400 \mathrm{~K}}$ of $\mathrm{SrCu}_{3} \mathrm{Fe}_{4} \mathrm{O}_{12}$ measured by means of SXRD (Ref. 6, heating), XRD (heating), TMA (cooling), and SG (heating). (b) Linear thermal expansion $\mathrm{d} L / L_{400 \mathrm{~K}}$ of $\mathrm{SrCu}_{3} \mathrm{Fe}_{4} \mathrm{O}_{12}, \mathrm{CuO}$, and $\alpha-\mathrm{Fe}_{2} \mathrm{O}_{3}$ phases of the sample. The lines represent the linear fitting results. (c) Linear thermal expansion $\mathrm{d} L / L_{300 \mathrm{~K}}$ of $\mathrm{SrCu}_{3} \mathrm{Fe}_{4} \mathrm{O}_{12}$ measured using strain gauge upon cooling and heating. 
and SG methods involve measurements on the gross CTE of the sample pellet, which includes the impurity phases with a positive CTE. The Rietveld refinement results gave a positive CTE for $\mathrm{CuO}\left(5.5 \times 10^{-6} \mathrm{~K}^{-1}\right)$ and $\alpha-\mathrm{Fe}_{2} \mathrm{O}_{3}\left(6.8 \times 10^{-6} \mathrm{~K}^{-1}\right)$ in the temperature range $200-300 \mathrm{~K}$ [Fig. 3(b)]. These components and the unidentified phases significantly suppress the bulk NTE. The bulk CTE of the sample was calculated to ca. $-1.5 \times 10^{-5} \mathrm{~K}^{-1}$, in accordance with the formula: $\alpha=\left(\sum_{i}^{n} \alpha_{i} E_{i} V_{i}\right) /\left(\sum_{i}^{n} E_{i} V_{i}\right)$, where $\alpha_{i}, E_{i}$, and $V_{i}$ represent CTE, Young's modulus, and volume fraction for the $i$-th component, respectively, and $E_{i}$ for all the components were assumed to the same value. This CTE value remains larger than those obtained from the TMA and SG data. One of the reasons is the simple assumption of the Young's modulus. Another possible reasons are the sinterability, grain boundary, and porosity of the sample, as pointed out in the case of $(\mathrm{Bi}, \mathrm{La}) \mathrm{NiO}_{3}$. ${ }^{4}$

Figure 3(c) shows the temperature dependence of the linear thermal expansion $\mathrm{d} L / L_{300 \mathrm{~K}}$ of SCFO upon cooling and heating, as determined from the SG measurements. In both the cooling and heating processes, almost identical NTE behaviors were observed, confirming that the NTE of SCFO is reversible. Furthermore, the thermal hysteresis was negligible $(\sim 2.5 \mathrm{~K})$ in the NTE temperature range. This was because the NTE of SCFO was attributed to the second-order phase transition, as indicated by XRD.

The above results may augment the advantages of SCFO in practical applications, although the sample preparation process is complex. Since SCFO retains a cubic symmetry over a wide temperature range of $80-450 \mathrm{~K}$, isotropic expansion and contraction can be expected. In addition, the thermal hysteresis of SCFO is considerably small. These properties may help in preventing the generation of microcracks during thermal cycles. Further modification such as chemical substitution may improve the NTE characteristics of this compound.

\section{Summary}

Dilatometric analysis for the direct observation of the negative thermal expansion in $\mathrm{SrCu}_{3} \mathrm{Fe}_{4} \mathrm{O}_{12}$ was performed by using a thermomechanical analyzer and strain gauge. Reversibility and small thermal hysteresis in the negative thermal expansion temperature range were observed, leading us to conclude that the negative thermal expansion of $\mathrm{SrCu}_{3} \mathrm{Fe}_{4} \mathrm{O}_{12}$ is attributed to second-order phase transition.

Acknowledgements A part of this study was performed using the facilities at the Materials and Structures Laboratory, Tokyo Institute of Technology. The synchrotron radiation experiments were performed at SPring-8 with the approval of the Japan Synchrotron Radiation Research Institute (JASRI) (Proposal No. 2013A1043). This work was partly supported by Nippon Sheet Glass Foundation for Materials Science and Engineering.

\section{References}

1) J. S. O. Evans, Dalton Trans., 1999, 3317-3326 (1999).

2) K. Takenaka, Sci. Technol. Adv. Mater., 13, 013001 (2012).

3) K. Takenaka and H. Takagi, Appl. Phys. Lett., 87, 261902 (2005).

4) M. Azuma, W. T. Chen, H. Seki, M. Czapski, S. Olga, K. Oka, M. Mizumaki, T. Watanuki, N. Ishimatsu, N. Kawamura, S. Ishiwata, M. G. Tucker, Y. Shimakawa and J. P. Attfield, Nat. Commun., 2, 347 (2011).

5) I. Yamada, K. Takata, N. Hayashi, S. Shinohara, M. Azuma, S. Mori, S. Muranaka, Y. Shimakawa and M. Takano, Angew. Chem., Int. Ed., 47, 7032-7035 (2008).

6) I. Yamada, K. Tsuchida, K. Ohgushi, N. Hayashi, J. Kim, N. Tsuji, R. Takahashi, M. Matsushita, N. Nishiyama, T. Inoue, T. Irifune, K. Kato, M. Takata and M. Takano, Angew. Chem., Int. $E d ., 50,6579-6582$ (2011).

7) Y. W. Long, N. Hayashi, T. Saito, M. Azuma, S. Muranaka and Y. Shimakawa, Nature, 458, 60-63 (2009).

8) H. Etani, I. Yamada, K. Ohgushi, N. Hayashi, Y. Kusano, M. Mizumaki, J. Kim, N. Tsuji, R. Takahashi, N. Nishiyama, T. Inoue, T. Irifune and M. Takano, J. Am. Chem. Soc., 135, 6100-6106 (2013).

9) The crystal structure was drawn using the program VESTA: K. Momma and F. Izumi, J. Appl. Cryst., 41, 653-658 (2008).

10) F. Izumi and K. Momma, Solid State Phenom., 130, 15-20 (2007). 